\title{
Management of anaemia and blood transfusion in critical care - implementing national guidelines in ICU
}

\author{
Sethina Watson, Kate Kendrick
}

North Bristol Trust

\begin{abstract}
Anaemia in intensive care is common, with approximately $50 \%$ of patients receiving a red cell transfusion. Recognised complications from transfusion include 'transfusion associated lung injury', infection, and organ failure progression. Most cohort studies show a positive relationship between red cell transfusion and adverse outcomes. In 2012, the British Committee for Standards in Haematology issued guidelines for red cell (RBC) transfusion in critical care. They recommend a haemoglobin transfusion trigger of below $70 \mathrm{~g} / \mathrm{dL}$ unless the patient is bleeding, has acute sepsis, neurological injury, or an acute coronary syndrome.
\end{abstract}

RBC transfusions in a single intensive care unit (ICU) were prospectively assessed for compliance with national guidance. Each transfusion was categorised with a traffic light system: red for inappropriate, green for appropriate, and amber for those that were not clearly appropriate or inappropriate.

The quality improvement project began with a clinical effectiveness audit of doctors' knowledge of critical care transfusion thresholds. Two quality improvement interventions were used: 1) a local blood transfusion guideline was produced and posters were placed in the ICU 2) this guidance was attached to the transfusion prescriptions. Data was collected after each intervention. A total of 30 random adult RBC transfusions were analysed between August 2013 and February 2014.

Despite good results from the effectiveness audit an assessment of RBC transfusions demonstrated room for improvement. Prior to introduction of the guideline intervention, a total of two transfusions were green, one red and seven amber. Following both interventions there were seven green transfusions and three amber. No transfusions were classed as inappropriate. According to additional trust based ICU transfusion records, there was approximately a $50 \%$ reduction ( 41 to $18 \mathrm{RBC}$ transfusions) in overall blood transfusions following the first intervention in October 2013.

Simple interventions to raise awareness such as surveys, posters, and reminders can dramatically improve RBC prescribing in accordance to evidence based guidelines. Making prescribers aware of guidelines can dramatically reduce the total number of overall transfusions and increase transfusion appropriateness.

\section{Problem}

Inappropriate blood transfusions can cause harm and sometimes worsen patient outcomes. Blood transfusions are common, yet the decision to transfuse is complex. In the intensive care setting, the decision whether to prescribe blood products in anaemic patients without major haemorrhage can be further complicated by the patient's co-morbidities. Good clinical judgement is required and individual risk and circumstances play a key part in the decision making process. The use of evidence based guidelines can aid the clinician's decision and improve patient safety.

In addition to the more common risks of transfusion (administration errors, contamination and so on) 'transfusion associated lung injury' (TRALI) and 'transfusion associated circulatory overload' (TACO) are relevant complications for the critical care patient. Many cohort studies show an association between transfusion and adverse patient outcomes including death, organ failure, infection, and extended hospital stay (study references are detailed in the $\mathrm{BCSH}$ guidelines).
The aim of this quality improvement project is to improve the prescription of blood transfusion products for intensive care patients by educating doctors on evidence based transfusion guidelines and providing useful implementation tools for quality improvement. Producing locally appropriate guideline based interventions for use on the ward will aid the doctor in the decision making process. It was conducted at a nine-bedded intensive care unit (ICU) at Southmead Hospital, Bristol, UK.

\section{Background}

The British Committee for Standards in Haematology (BCSH) issued guidelines in 2012 for the use of red cell transfusion in critically ill patients. Designed for day to day use by the treating physician they offer advice on how to manage anaemia when major haemorrhage is not present. This project investigated at what $\mathrm{Hb}$ level RBC transfusions were triggered in critical care patients and determined whether these transfusions adhered to recommended guidelines. We developed a local guideline implementation strategy based on these BCSH guidelines for North Bristol Trust, 
Southmead Hospital ICU. Southmead ICU/HDU is a nine-bedded unit and our guidelines were designed for use by all ICU doctors from foundation trainees to consultants. The Trust's Frenchay site has 17 further ICU/HDU beds.

Some basic facts are as follows:

The World Health Organisation defines anaemia in men and women as a haemoglobin $(\mathrm{Hb})$ of $<130$ and $<120 \mathrm{~g} / \mathrm{L}$ respectively. Severe anaemia is an $\mathrm{Hb}$ of $<80 \mathrm{~g} / \mathrm{L}$.

Key components of the guidelines are:

A transfusion threshold of $70 \mathrm{~g} / \mathrm{L}$ or below, with a target haemoglobin $(\mathrm{Hb})$ range of $70-90 \mathrm{~g} / \mathrm{L}$, should be the default for all critically ill patients, unless specific co-morbidities or acute illness related factors modify clinical decision making. Transfusion triggers should not exceed $90 \mathrm{~g} / \mathrm{L}$ in most critically ill patients. Patients with ischaemic heart disease, sepsis, and neurological conditions have differing transfusion thresholds.

\section{Baseline measurement}

We began by assessing ICU doctors' knowledge of appropriate ICU blood transfusion practices with a clinical effectiveness audit. All doctors working in ICU at North Bristol Trust were invited to reply to an online email survey. The number invited included all ICU consultants $(n=25)$ and all NBT ICU/anaesthetic trainees including senior trainees $(n=59)$, a total of 84 . Of the total of 84,38 doctors responded to the initial survey. Over $94 \%$ of ICU doctor respondents had good knowledge of appropriate evidence base and correctly identified a transfusion trigger of $70 \mathrm{~g} / \mathrm{L}$ in critically ill patients without specific co-morbidities. The $\mathrm{BCSH}$ guideline also states that transfusion triggers should not exceed $90 \mathrm{~g} / \mathrm{L}$ in most critically ill patients.

We asked questions for the following co-morbidities and transfusion strategies. For severe sepsis with clear evidence of insufficient global oxygen delivery, $84 \%$ of respondents correctly chose a transfusion target $\mathrm{Hb}$ of $90-100 \mathrm{~g} / \mathrm{L}$. However, there was room for improvement specifically for ACS transfusion triggers. There was some confusion over the appropriate target triggers and $\mathrm{Hb}$ for patients with stable angina. When cross tabulated by grade consultants (nine of 20) were more likely to consider transfusing at $>80-90 \mathrm{~g} / \mathrm{L}$. The $\mathrm{BCSH}$ guidelines recommend maintaining an $\mathrm{Hb}$ of $>70 \mathrm{~g} / \mathrm{L}$ for this patient group, only $15 \%$ of respondents chose this target. Respondents knew that red cell transfusion should not be used as a strategy to assist weaning from mechanical ventilation when the $\mathrm{Hb}$ is $>70 \mathrm{~g} / \mathrm{L}$ ( $84 \%$ of doctors knew this). Overall, $86 \%$ knew that a more liberal blood transfusion trigger of $\mathrm{Hb}<100 \mathrm{~g} / \mathrm{L}$ is associated with poorer outcomes in morbidity and mortality.

The second stage in the baseline measurement was to determine whether this knowledge translated to prescribing practice. A clinical audit was completed to assess red blood cell (RBC) prescriptions for any adult patient in ICU that did not have a major haemorrhage. We audited $10 \mathrm{RBC}$ transfusions and compared them with the BCSH 2012 guideline.
Data collection included patient age, gender and co-morbidities, as well as the ICU diagnosis, reason for transfusion (if given on the prescription), and $\mathrm{Hb}$ pre and post transfusion. Using this information, transfusions were categorized into one of three groups:

- Green: this was used for appropriate transfusions. For example, $\mathrm{Hb}<70 \mathrm{~g} / \mathrm{L}$ or $<80 \mathrm{~g} / \mathrm{L}$ if the patient had other co-morbidities (usually ACS or acute sepsis)

- Red: this was used for inappropriate transfusions. Any transfusions that occurred with a $\mathrm{Hb}$ of $>90 \mathrm{~g} / \mathrm{L}$ were immediately placed in this category

- Amber: for borderline transfusions which required more clinical information in order to make a decision.

This was used for transfusions which did not fit in to either the green or red areas. An example of an amber transfusion is the patient that follows.

A 68 year old female with myelodysplasia resulting in neutropenia. This patient was transfused at a $\mathrm{Hb}$ of $73 \mathrm{~g} / \mathrm{L}$. While this does not fall in to the green category ( $\mathrm{as} \mathrm{Hb}$ is $>70$ ), we did not feel that it deserved to go in the red category. Instead it fits into the amber category, requiring more clinical judgement. A transfusion like this is at the discretion of the consultant using more information than we collected, including how unwell the patient was and which way the $\mathrm{Hb}$ was trending. We noted many transfusions were given when the $\mathrm{Hb}$ was trending downwards, and instead of waiting until the $<70$ $\mathrm{g} / \mathrm{L}$ mark, transfusions were given when $\mathrm{Hb}$ was in the low 70's. While these may technically be red (inappropriate) transfusions, we allowed a little more flexibility with our data and thus had an amber category.

The results showed that only $2 / 10$ of transfusions were appropriate (coded green) when compared with the guidelines, 7/10 were borderline (amber), and 1/10 were inappropriate (coded red.) This data demonstrated significant knowledge gaps in appropriate transfusions practices for ICU patients.

As well as looking at each transfusion to determine if it was appropriate, we also assessed if there were certain conditions that were common to be inappropriately prescribed. In the first data collection both green transfusions occurred at an $\mathrm{Hb}$ of $<70 \mathrm{~g} / \mathrm{L}$. The amber data consisted of three ACS patients and four other patients, and the inappropriate transfusion was an ACS transfused an $\mathrm{Hb}$ of $104 \mathrm{~g} / \mathrm{L}$. Patients admitted to this ICU ranged from those with sepsis to those with vascular, renal orthopaedic, urological, and general surgical needs. Analysing patient sub-groups did not identify one specific group as more likely to have red, amber, or green prescribing.

We developed an NBT ICU blood transfusion guideline based on BCSH recommendations.

See supplementary file: ds2966.docx - "ICU Hb Targets Guide1.4"

\section{Design}




\section{BMJ Quality Improvement Reports}

The first intervention consisted of making the guidelines into a poster which was displayed in the doctors hub on the ICU. The guideline was patient centric and used a flow-chart style, assisting doctors in making the decision whether to transfuse with blood products. A copy of the guideline is included in this report.

Following the implementation of the guideline, data collection of 10 transfusions was performed. This data was then analysed with comparison to transfusions carried out before the guidelines were made available.

The second intervention also consisted of using the flowchart with the guidelines. Although the guidelines were already present in poster form on the ICU, we decided to make a smaller version and attach them to the blood transfusion prescription forms. This means that in order for a blood transfusion to be prescribed, the doctor has to look at the guidelines.

Following this intervention data from a further 10 transfusions were collected, and the data analysed.

\section{Strategy}

Cycle 1

Plan - Assess staff knowledge of blood transfusion guidelines

Do - Send email survey to all ICU doctors in two hospitals

Study - Results will identify knowledge base and provide answers to important components of the guideline

Act - Determine knowledge gaps and in preparation for creating guide to blood transfusion in critical care

Cycle 2

Plan - Audit 10 patients who have received blood transfusions prior to any intervention to assess baseline data

Do - Use patient notes and blood results to assess transfusion triggers and indication for transfusion

Study - Did transfusions follow national guidelines?

Act - Produce guidelines to aid transfusion prescribing

Cycle 3

Plan - Develop guidelines for use in critical care setting

Do - Display the guidelines in poster form on the ICU, and email a copy to the doctors to make them aware. Collect a further 10 sets of data to see if there has been an improvement

Study - Analyse results of appropriate transfusions
Act - Improve guideline for real-time use to encourage best transfusion practices, and consider need for further intervention to make the guidelines more available

Cycle 4

Plan - Develop small A5 sized transfusion guidelines for use in critical care setting

Do - Attach smaller transfusion guideline to blood prescription form seen by all doctors prescribing blood products. Collect a further 10 sets of data to see if there has been a further improvement

Study - Analyse results of appropriate transfusions

Act - Improve guideline for real-time use to encourage best transfusion practices. Consider re-auditing in two to four months to assess if improvement has been sustained

\section{Post-measurement}

The first Plan Do Study Act (PDSA) cycle was a clinical effectiveness audit of all ICU doctors' knowledge of appropriate haemoglobin thresholds to trigger transfusion. Following these results a clinical audit was deemed necessary and local ICU transfusion guidelines were developed.

The second PDSA cycle was completed before the development of the guidelines and involved auditing patient notes. Our baseline measurements showed only 2/10 RBC transfusions were appropriately prescribed according to the guidelines. A colour A4 sized guideline poster was then developed in a flow chart style to aid real time decision making based on clinical picture and comorbidities. This was displayed in the Southmead ICU staff working hub. Several copies were placed in view of all doctors (and other staff working on the unit).

PDSA cycle 3 was completed and we re-audited another 10 RBC transfusions. The results showed significant improvements: 4/10 transfusions were appropriate (green), $5 / 10$ borderline (amber), and $1 / 10$ were inappropriate (red).

For the second data set, one of the appropriate transfusions occurred $<70 \mathrm{~g} / \mathrm{L}$, and another three were patients transfused at $<80 \mathrm{~g} / \mathrm{L}$ with ACS. All five of the amber transfusions did not have any of the additional risks (such as ACS, sepsis or neurological injury). The one red transfusion was again an ACS transfused at $>90 \mathrm{~g} / \mathrm{L}$.

With this improvement in prescribing thresholds, we decided to produce a smaller A5 colour version of the guideline and attach it to the blood transfusion prescription. Therefore, any doctor prescribing a RBC transfusion would encounter the guideline. With this intervention a further $10 \mathrm{RBC}$ transfusions were audited (PDSA cycle four). Following both interventions results showed $7 / 10$ transfusions were appropriate (green), 3/10 were borderline (amber), and none were inappropriate (red). 
Our final transfusion data comprised of three green ACS transfusions, but another two amber ACS transfusions. We had two green transfusions with sepsis, and another two where the $\mathrm{Hb}<70$ $\mathrm{g} / \mathrm{L}$. Generally it appears that the transfusion trigger of $<70 \mathrm{~g} / \mathrm{L}$ is easily understood and acted upon, but there still seems to be some misunderstanding about patients who have higher transfusion triggers, especially ACS. This would be an area to target in further audits.

For confirmation of our findings we asked the Trust's blood transfusion lead manager to analyse RBC transfusions for patient's in the ICU. Comparing six weeks prior to the introduction of the A4 poster and six weeks after there was around a $50 \%$ reduction of ICU RBC transfusions (a reduction of 41 to 18 RBC units transfused).

To maintain these significant improvements a further intervention has been conducted in Feb 2014. This was an educational email sharing positive results and introducing guidelines to new trainees arriving in the unit. Currently, another 10 random RBC transfusions are being audited.

See supplementary file: ds3137.docx - "Graphs showing the improvement in appropriate blood transfusions post interventions"

\section{Lessons and limitations}

Our initial clinical effectiveness audit seemed to suggest that doctors already knew appropriate ICU transfusion triggers. However, our first PDSA cycle prior to intervention showed poor practical application of knowledge. When creating the guideline it needed to be quick to read, easy to follow, and include additional information to assist in the doctors' decision making. Adapting a national guideline for local use in a busy ICU environment required modifications in style and content. We found the BCSH algorithm presented the guideline in the most appropriate format, so we made only a few modifications. The guideline used a flow chart format in initially muted colours which the feedback suggested that this was not bright enough, so we changed this to bright primary colours instead. We engaged key leads in Trust blood transfusion during our guideline development. Additionally, the content of the guideline was discussed by ICU consultants and the trust, and a local change was made for neurological patients. We tested four different versions of the guideline until settling on the current version. We then established a review date.

Engaging ICU staff required communication of our project's aims and intentions, as well as sharing the results of their improvement in prescribing practice. As the project progressed and the number of transfusions reduced significantly the time it took to collect data grew longer thus lengthening the project completion time. Two foundation doctors collected the audit data and the ability to collect data depended on differing rotas and changing jobs or sites. This meant that sometimes we were unable to collect audit data and patients who had been transfused had been discharged. Our sample size of 10 random RBC transfusions between interventions may seem small, but this was an appropriate size in a small nine bed ICU unit. We initially selected patients while on ICU, but this missed patients who may have only been in ICU for a day or so. But using Trust transfusion documentation we were able to follow up patients on wards once discharged from ICU.

Our results were unexpected, both at the outset in the number of inappropriate transfusions, and by PDSA cycle three demonstrating significant improvements in prescribing. Although statistical analysis using Fisher's exact test has shown that the improvement in transfusions was not statistically significant, we hope that with further auditing and analysis of a larger data set would show such statistical significance. It is worth noting that although our numbers per cycle are small (10), there are only an average of 23 transfusions per month in this nine bedded ICU, meaning our data captures a good proportion of these transfusions.

Sustaining the improvement in RBC prescribing is an ongoing challenge especially when trainees shift hospitals every few months. A new intake of SHOs has arrived and has already been updated on BCSH guidelines. Subsequent PDSA cycles are planned to monitor practices. Future revisions to the guideline shall be made pending results of audit and release of new evidence based data. An online e-learning tool may be developed with case based transfusion decisions for doctors in training. The guideline has been incorporated into Trust transfusion documents and a similar project is planned at the another ICU location. There is a planned hospital site merge where two NBT ICUs are located in the new Southmead Hospital. Once merged the guideline may then be modified for use in a single 48 bed ICU, presenting further challenges in designing, implementing, and sharing local guidelines.

\section{Conclusion}

This project successfully demonstrated that nationally written evidence based guidelines can be locally adapted with good outcomes in RBC prescribing practices. We initially determined whether knowledge was sufficient to not need any guideline. Despite most doctors knowing appropriate parameters for transfusing critical care patients there were demonstrated gaps between knowledge and prescribing practice. Before introducing our guideline, doctors may have erred towards transfusion when faced with complex patients with diverse co-morbidities. Highlighting to ICU doctors that different co-morbidities have unique transfusion triggers and that their recommended target $\mathrm{Hb}$ may be lower than thought is one explanation for the dramatic drop in RBCs prescribed. The guideline provided prescribing doctors with bedside information on the appropriateness of transfusion, prompting individual reflection or team discussion on a patient's clinical need. Having evidence based targets readily available during the clinical decision making process may have contributed to a more conservative transfusion strategy as doctors felt more comfortable tolerating lower patient $\mathrm{Hb}$ levels.

At present there exists no clinically or cost effective alternative for restoring $\mathrm{Hb}$ levels in the critically ill patient. A unit of blood costs around £122, plus the costs associated with equipment, staff, monitoring, investigations, hospital stay and any adverse outcomes. Out trust's transfusion data shows an average of 63 RBC units prescribed per month across its two main ICUs. We estimate that if 


\section{BMJ Quality Improvement Reports}

sustained our $50 \%$ reduction in RBCs prescribed could save North Bristol Trust around £46,116 per annum.

Reducing the number of inappropriate and borderline transfusions from $80 \%$ to $30 \%$ reduces patient risk of adverse transfusion outcomes such as TRALI and TACO, as well as transfusion reactions, formation of antibodies, and so on. As the project progresses the need to include data on patient outcomes is paramount to truly measure the success of appropriate RBC prescribing. Presently, this work is outside the parameters of our QI project. In the future, further audit and research into morbidity and mortality prior to and following the guideline introduction would provide key information on patient outcomes. Sustaining the reduction will require ongoing work to highlight evidence based data; work that may be both creative and cost effective. The balance of risks and benefits associated with RBC transfusions is an ongoing challenge for both prescribers and patients. Simple tools to aid clinical decisions can be invaluable.

\section{References}

1. Retter, A., Wyncoll, D., Pearse, R., Carson, D., McKechnie, S., Stanworth, S., et al for the British Committee for Standards in Haematology 2013 Guidelines on the management of anaemia and red cell transfusion in adult critically ill patients. British Journal of Haematology, 160, 445-64.

2. Szczepiorkowski, Z., Dunbar, N. Transfusion guidelines: when to transfuse, Hematology, 2013, 1, 638.

3. NHS Blood and Transplant Annual Review 2012-2013 [Internet]. 2014 Feb 18. Available from: http://www.nhsbt.nhs.uk/annualreview/blood-supply/

\section{Declaration of interests}

Nothing to declare.

\section{Acknowledgements}

Jasmeet Soar, Janet Birchall, Matt Thomas, Tim Wreford-Bush, Thomas Milne. 